\title{
Gabapentina a dosis de 300 vs. 450 mg como premedicación anestésica para hipertensión reactiva, ansiedad y analgesia
}

Dulce María Rascón-Martínez¹, Joaquín Antonio Guzmán-Sánchez1, Nora Paulina Corral-Urdapilleta1, Gema Damaris Arguelles-Uribe', Jazmín Velázquez-Loeza1, Gustavo Soto-Palma y Orlando Carrillo-Torres² ${ }^{1}$ Departamento de Anestesiología, Unidad Médica de Alta Especialidad, Hospital de Especialidades, Centro Médico Nacional Siglo XXI, Instituto Mexicano del Seguro Social; ${ }^{2}$ Departamento de Anestesiología, Hospital General de México Dr. Eduardo Liceaga. Ciudad de México, México

\section{Resumen}

Objetivo: Observar el comportamiento de la gabapentina para aminorar la hipertensión reactiva secundaria a ansiedad y dolor en pacientes sometidos a cirugía oftálmica, así como el consumo de opiáceos entre los grupos. Método: Ensayo clínico controlado aleatorizado y doble ciego que analizó a 125 pacientes divididos en tres grupos: grupo A, gabapentina 300 mg; grupo B, gabapentina $450 \mathrm{mg}$; grupo C, amaranto en grageas como control 2 horas antes del procedimiento quirúrgico. Se utilizó la prueba de ji al cuadrado para variables sociodemográficas y ANOVA de un factor para variables numéricas continuas. Se consideró como significativo un valor de $p<0.05$ para un estudio de dos colas con un poder beta del $80 \%$. Resultados: La ansiedad y la analgesia transoperatoria y posoperatoria tuvieron diferencias significativas entre los grupos. Se encontró menor consumo de opiáceos en los grupos que usaron gabapentina. Conclusiones: La gabapentina por vía oral, 300 o $450 \mathrm{mg}$, 2 horas antes de la cirugía, reduce el dolor, la ansiedad y el consumo de opiáceos durante el posoperatorio en pacientes sometidos a cirugía oftalmológica.

PALABRAS CLAVE: Gabapentina. Hipertensión reactiva. Ansiólisis. Analgesia.

\begin{abstract}
Objective: Observe the behavior of gabapentin to reduce reactive hypertension secondary to anxiety and pain in patients undergoing ophthalmic surgery as well as opioid consumption between groups. Method: Clinical randomized double blind controlled trial that analyzed 125 patients divided into three groups: Group A, gabapentin $300 \mathrm{mg}$; Group B, gabapentin 450 $\mathrm{mg}$; and Group $C$, amaranth dragees as a control $2 \mathrm{~h}$ before the surgical procedure. Chi-squared test was used in sociodemographic variables and one-way ANOVA for continuous numeric variables. It was considered as significant a $p<0.05$ for a study of two tails with a power of $80 \%$ beta. Results: Anxiety and analgesia intraoperative and postoperative had significant differences between groups. Lower consumption of opioid was found in the groups that used gabapentin. Conclusions: Gabapentin orally 300 or $450 \mathrm{mg}$ h prior to surgery reduces pain, anxiety and postoperative opioid consumption in patients undergoing ophthalmic surgery.
\end{abstract}

KEY WORDS: Gabapentin. Reactive hypertension. Anxiolysis. Analgesia.

\author{
Correspondencia: \\ Orlando Carrillo-Torres \\ Pestalozzi, 38 \\ Dep. 1, Col. Piedad Narvarte, Del. Benito Juárez \\ C.P. 03000 , Ciudad de México, México \\ E-mail: orlo_78@hotmail.com
}

Fecha de recepción en versión modificada: 28-08-2016

Fecha de aceptación: 01-12-2016

DOI://dx.doi.org/10.24875/GMM.17002796
Gac Med Mex. 2018;154:54-61

Contents available at PubMed www.gacetamedicademexico.com 


\section{Introducción}

La ansiedad, el dolor y la reactividad cardiovascular son factores que desempeñan un papel en la hipertensión sostenida. Aunque los ansiolíticos carecen de una respuesta significativa sobre la hipertensión relacionada con estrés crónico, sí funcionan en la hipertensión aguda de origen reactivo debido a que los trastornos de ansiedad crónica tienden a caracterizarse por la prevalencia de hipertensión secundaria a estímulos externos, independientemente de la respuesta autónoma ${ }^{1}$.

La ansiedad en el contexto hospitalario se describe como un estado desagradable de malestar o tensión emocional, de carácter secundario, generado por la preocupación variada del paciente respecto a la enfermedad, la hospitalización, la anestesia y el evento quirúrgico ${ }^{2}$. La incidencia de la ansiedad preoperatoria en los adultos varía del 11 al $80 \%$, dependiendo de si la medición se realiza mediante cuestionarios 0 mediante parámetros clínicos ${ }^{3}$.

En el contexto quirúrgico, la ansiedad, llamada también ansiedad perioperatoria, está influenciada por la percepción del paciente sobre su salud en general, la incertidumbre sobre el futuro, el tipo de cirugía, la anestesia a realizar, las molestias posoperatorias, el dolor y hasta el temor a la muerte 4 , siendo una característica constitucional de la personalidad del paciente combinada con el entorno del acto quirúrgico ${ }^{5}$.

Los pacientes que presentan ansiedad en el preoperatorio tienen un riesgo estimado mayor de 2.6 veces en el período posoperatorio 6 . Un alto grado de ansiedad, a su vez, se asocia a mayor dolor, aumento en la demanda de analgésicos y poca satisfacción ${ }^{7}$.

Existen diversos tipos de ansiedad, pero la que se genera en el paciente por el acto anestésico-quirúrgico es un malestar psíquico y físico que nace de la sensación de peligro inmediato, y se caracteriza por temor difuso, que puede fluctuar de la inquietud al pánico ${ }^{8}$.

Durante el transanestésico, un paciente ansioso responde de diferente manera que un paciente tranquilo. Los requerimientos de los agentes de inducción tienden a incrementarse en los pacientes ansiosos ${ }^{9} \mathrm{y}$ existe predisposición a fluctuaciones hemodinámicas secundarias a reacciones autonómicas en la fase de mantenimiento.

Izurieta y Jeffrey ${ }^{10}$ observaron alteraciones en pacientes ansiosos hospitalizados, como disfunción pulmonar, agitación y síndrome de estrés postraumático de tipo neurosis.
La ansiedad es una sensación emocional subjetiva y desagradable, por lo que realizar una evaluación de la misma se torna difícil ${ }^{11}$. Para poder tratarla adecuadamente hay que hacer una evaluación objetiva. Esta cuantificación puede llevarse a cabo con escalas que permiten hacer una valoración inicial y comprobar el efecto de los tratamientos administrados. Para ello existen diversas escalas, tales como la STAI (State-Trait Anxiety Inventory Questionnaire), la escala visual análoga de ansiedad, las escalas de ansiedad de Taylor (Inventario de Situaciones y Respuestas de Ansiedad) o Hamilton, y más recientemente la Escala de Ansiedad Preoperatoria y de Información de Ámsterdam (APAIS).

La APAIS consiste en una serie de seis preguntas con un valor de 1 a 5 puntos para cada respuesta; la puntuación máxima es de 20 puntos. Cuenta con una subescala de información con un valor de 1 a 5 puntos para cada respuesta, en la que obtener una puntuación entre 5 y 7 indica que los pacientes no requieren información; una calificación entre 8 y 10 indica que los pacientes requieren información más completa ${ }^{12}$.

La ansiedad perioperatoria supone un reto para todo anestesiólogo. En los últimos años se ha propuesto a la gabapentina durante el preoperatorio como una buena opción con pocos efectos secundarios ${ }^{13}$.

El papel de la gabapentina ha sido estudiado en diversas situaciones, como analgesia posoperatoria, prevención del dolor crónico posquirúrgico, atenuación de la respuesta hemodinámica a la laringoscopia y la intubación, y prevención de las náuseas, los vómitos, el delirio posoperatorio y la ansiólisis preoperatoria. Diversos estudios reflejan áreas importantes de investigación en anestesia y señalan el papel importante de un solo medicamento con efecto multimodal ${ }^{14-16}$.

La gabapentina fue introducida en 1993 como anticonvulsivante, adyuvante para el tratamiento de las crisis parciales refractarias. Posteriormente se demostró su eficacia en una variedad de condiciones de dolor crónico, incluyendo la neuralgia posherpética, la neuropatía diabética, el síndrome de dolor regional complejo, el dolor inflamatorio, el dolor central, el dolor de origen maligno, la neuralgia del trigémino, la neuropatía relacionada con el virus de la inmunodeficiencia humana y la cefalea ${ }^{17}$. En 2002, la gabapentina fue aprobada por la Food and Drug Administration de los EE.UU. para el tratamiento de la neuralgia posherpética. En el Reino Unido, la gabapentina tiene una completa licencia para el tratamiento de todos los tipos de dolor neuropático. El uso se ha extendido más recientemente a la gestión de condiciones más 
agudas, en particular en el período perioperatorio. Hasta ahora se han documentado las posibles y diversas funciones de la gabapentina en más de 30 ensayos clínicos ${ }^{18,19}$.

La gabapentina es un análogo estructural del neurotransmisor ácido gamma-aminobutírico (GABA), cuya formula molecular es $\mathrm{C} 9 \mathrm{H} 17 \mathrm{NO} 2$ y tiene un peso molecular de 171.24. Es un cristal solido blanco altamente cargado a pH fisiológico, libremente soluble en medios ácidos o básicos. La absorción intestinal es dependiente de la dosis debido a un mecanismo saturable en el intestino, con la biodisponibilidad variando inversamente con la dosis (luego de dosis de 300 y $600 \mathrm{mg}$ es del 60 y el $40 \%$, respectivamente). No se fija a las proteínas plasmáticas y tiene un volumen de distribución de 0.6-0.8 l/kg; el pico de concentración plasmática luego de una dosis de $300 \mathrm{mg}$ por vía oral se logra a las $2-3$ horas $(2.7 \mathrm{mg} / \mathrm{ml})$, y la concentración en el cerebro es del $80 \%$ con respecto a la plasmática ${ }^{20}$.

El efecto ansiolítico se logra mediante la unión a la proteína de la subunidad $\alpha 2-\delta$ de los canales de calcio dependientes del voltaje, funcionando como inhibidor de la liberación presináptica de varios neurotransmisores excitatorios ${ }^{21}$.

Se ha observado que una dosis única de gabapentina (300-1200 mg) administrada dentro de las 2 horas previas al inicio del evento quirúrgico disminuye el consumo de opiáceos en el transanestésico y el posanestésico, en un rango que oscila entre un 20 y un $62 \%$. Debido a que el metabolismo hepático y la unión a las proteínas es baja, no se conocen interacciones farmacológicas clínicamente relevantes ${ }^{22}$. En el estudio de Adam, et al. ${ }^{23}$, los efectos adversos que se observaron con mayor frecuencia fueron sedación y mareo.

Se ha demostrado que el uso preventivo de gabapentina a dosis de 300-1200 mg 2 horas antes de la cirugía mejora la analgesia postoperatoria y la movilización postoperatoria, al igual que disminuye el consumo de analgésicos en cirugía ortopédica de extremidades ${ }^{24-26}$.

Los estudios realizados hasta el momento apoyan el efecto de la gabapentina como ansiolítico y como coadyuvante del manejo de la hipertensión reactiva, aunque aún falta determinar cuál es la dosis más efectiva y con menos efectos adversos.

El objetivo fue observar el efecto de gabapentina para aminorar la hipertensión reactiva secundaria a ansiedad y dolor.

\section{Método}

Los sujetos fueron reclutados en el mismo hospital y fueron invitados a participar durante la visita preanestésica realizada previa al evento quirúrgico. Los pacientes fueron programados para cirugía correspondiente al área de oftalmología bajo anestesia regional (bloqueo retrobulbar). Se incluyeron pacientes catalogados como ASA I, II, y III según la clasificación de la American Society of Anaesthesiologist, programados para cirugía de catarata, vitrectomía o colocación de válvulas bajo sedación consciente, de entre 40 y 75 años de edad, que no hubieran recibido ansiolíticos, psicotrópicos ni neuromoduladores en los 3 días previos a la cirugía. Fueron excluidos los pacientes con contraindicaciones o alergias a gabapentina, demencia, delirium y deterioro cognitivo descartados por tamizaje, enfermedad renal crónica KDOQI IV o V, o insuficiencia hepática.

Se realizó un ensayo clínico prospectivo, longitudinal, aleatorizado y doble ciego. Se asignó a los integrantes de cada grupo mediante de una tabla de números aleatorios. La distribución de los medicamentos se realizó por un investigador externo, que no participó en las evaluaciones perioperatorias. Se dividió a la población en tres grupos y cada paciente recibió de manera aleatoria dos cápsulas de características iguales, al menos 2 horas antes de su ingreso al quirófano. Uno de los grupos (grupo A) recibió una cápsula de gabapentina de $300 \mathrm{mg}$ y una segunda cápsula sin medicamento. El segundo grupo (grupo B) recibió dos cápsulas de gabapentina de 300 y de 150 mg. El tercer grupo (grupo $C$, control) recibió dos cápsulas preparadas con amaranto. A todos los pacientes se les administró anestesia regional por medio de bloqueo retrobulbar previa sedación con midazolam y fentanilo. Al momento no existe un régimen estandarizado para la práctica de esta sedación, y dada la sinergia y el incremento de la sensibilidad del sistema nervioso central con ambas medicaciones, la sedación inicial se llevó a cabo con dosis mínimas de midazolam $(0.01 \mathrm{mg} / \mathrm{kg})$ y fentanilo $(1 \mu \mathrm{g} / \mathrm{kg})$.

A cada grupo se le evaluó el dolor y la ansiedad mediante una escala analógica numérica y la respuesta fue posteriormente degradada con escalas tipo Likert según la intensidad reportada.

En caso de ansiólisis inadecuada (cifras de presión arterial $>20 \%$ de la basal al comienzo de la cirugía) se preparó midazolam para administrar en dosis-respuesta hasta mantener unas cifras tensionales óptimas para el procedimiento. 
El tamaño de la muestra se seleccionó de acuerdo con el diseño del estudio, por lo que corresponde a un contraste de hipótesis de forma bilateral con una significación estadística de $p=0.05$ y un poder $\beta=0.80$. Se usó una fórmula que compara proporciones. Se tomaron como base reportes en los que existe una mejoría con gabapentina del $57 \%$ para la ansiólisis. El número resultante por grupo fue de 40 pacientes, aunque considerando un $10 \%$ de pérdidas se incrementó a 45 pacientes por grupo.

Para el análisis estadístico se utilizaron medidas de estadística descriptiva, como frecuencias simples y proporciones. Se utilizó la prueba de ji al cuadrado en variables sociodemográficas, y por el número de grupos se usó ANOVA de un factor como prueba paramétrica, o en su defecto la prueba de Kruskal Wallis de no contarse con una distribución normal en la variable. Se consideró como significativo un valor de $p<0.05$ para un estudio de dos colas con un poder $\beta$ del $80 \%$.

El estudio fue aprobado por los Comités de Ética y de Investigación del Hospital de Especialidades del
Centro Médico Nacional Siglo XXI del Instituto Mexicano del Seguro Social, con el número de registro N-2014-3601-30.

\section{Resultados}

Se incluyeron 135 pacientes, de los cuales se excluyó un 7.4\% ( $n=10)$; el 3.7\% $(n=5)$ pertenecían al grupo control, el $1.48 \%(n=2)$ al grupo de gabapentina $300 \mathrm{mg}$ y el $2.22 \%(n=3)$ al grupo de gabapentina $450 \mathrm{mg}$. En el $4.44 \%(n=6)$ de los casos, la cirugía fue suspendida por parte del servicio tratante debido a causas ajenas al estudio, y en el $2.96 \%$ $(n=4)$ de los casos se utilizó una técnica anestésica diferente a la propuesta. El análisis estadístico finalmente incluyo 125 pacientes (Fig. 1).

Del total de la muestra analizada, el $34.4 \%(n=43)$ fueron mujeres y el $65.6 \%(n=82)$ varones. La edad promedio fue de $59.71 \pm 13.03$ años. La descripción por variables clínico-demográficas se detalla en la tabla 1.

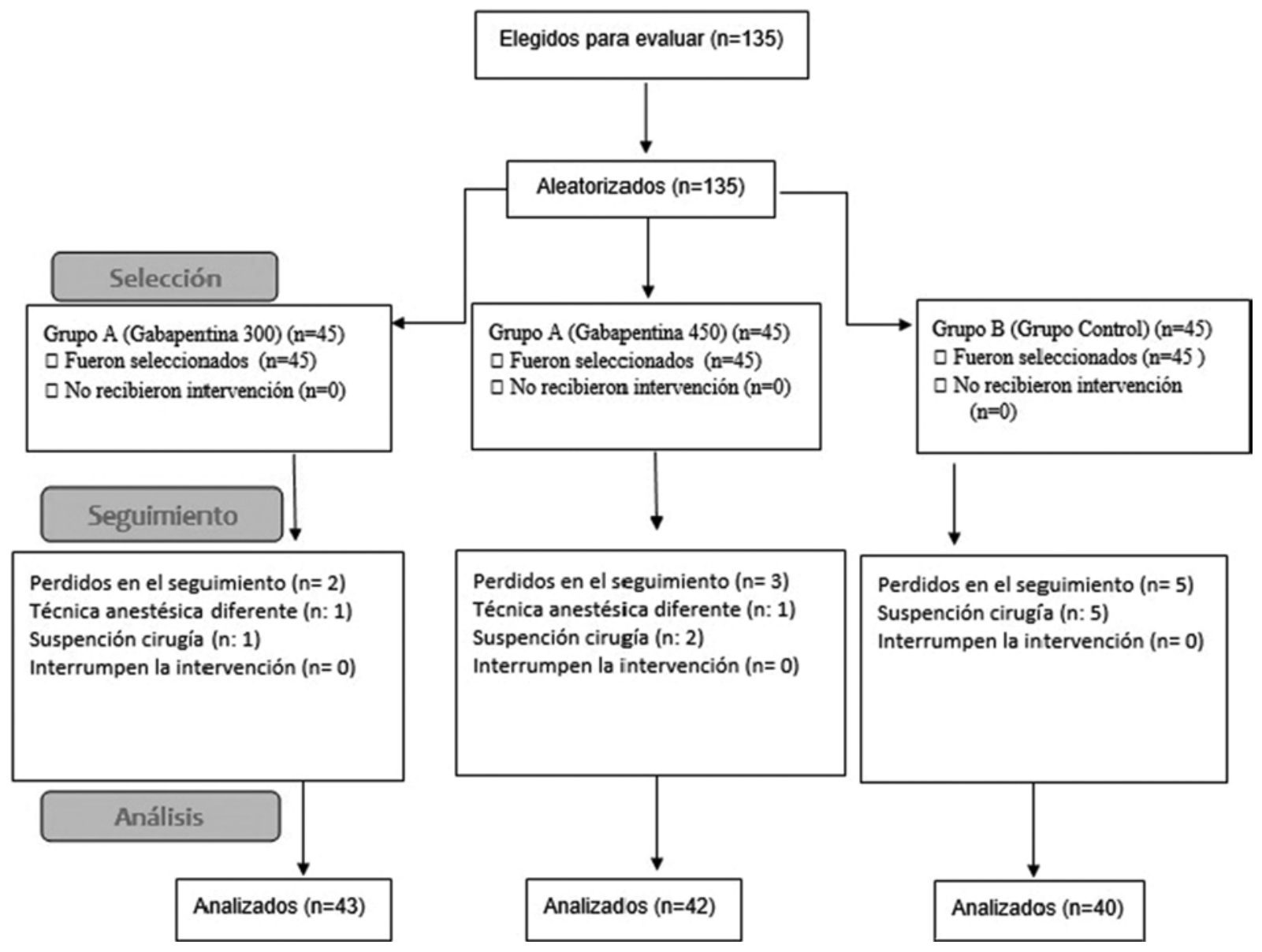


Para fines del estudio, la hipertensión reactiva fue definida como aquellos incrementos súbitos de la presión arterial por arriba del $20 \%$ de la basal (según los registros de enfermería), y que se presentara una disminución de la misma posterior a la aplicación de fármacos ansiolíticos. Del total de la muestra, el $57.6 \%$ ( $\mathrm{n}$ = 72) de los pacientes cumplió con la definición operacional del estudio. La distribución porcentual de pacientes que cursaron con hipertensión reactiva por grupos fue de la siguiente forma: grupo A, $20 \%(n=25)$; grupo B, 20.8\% $(n=26)$; y grupo C, $16.8 \%(n=21)\left(\chi^{2}=0.657\right.$; $\mathrm{gl}=2 ; \mathrm{p}=0.72)$.

La prueba de Kolmogórov-Smirnov mostró una distribución normal para variables hemodinámicas y respiratorias, con valores de $p>0.05$. Su comportamiento se detalla en la tabla 2.

\section{Ansiedad}

En nuestro estudio se analizó el grado de ansiedad mediante escalas tipo Likert en las que $0=$ nada, $1-3=$ poca, $4-6=$ moderada y 7-10 = fuerte. Las evaluaciones fueron realizadas en los siguientes momentos: la medición basal justo antes de la toma de la medicación en estudio, la segunda durante el monitoreo inicial en quirófano y finalmente en el posoperatorio inmediato. El análisis de esta variable se realizó mediante la prueba de Kruskal-Wallis, que arrojo un valor de $p=0.310$ para la ansiedad preoperatoria, $p=0.01$ para la ansiedad transoperatoria y $p=0.002$ para la ansiedad en el posoperatorio. Luego se realizó un análisis con la prueba de ji al cuadrado para ver con detalle la distribución porcentual de los grupos (Tabla 3).

Contrariamente, no se encontraron diferencias significativas en el consumo de midazolam total durante el procedimiento quirúrgico, para el que las medias reportadas fueron de $0.63 \pm 0.55 \mathrm{mg}$ (grupo A), $0.56 \pm 0.63 \mathrm{mg}$ (grupo B) y $0.74 \pm 0.55 \mathrm{mg}$ (grupo $C)(F=0.942$; $\mathrm{gl}=3 ; p=0.393)$.

\section{Dolor}

El dolor fue medido mediante una técnica clinimétrica usando la escala analógica numérica. De la misma forma, se dividió a la muestra de pacientes en aquellos sin dolor (0 puntos), con dolor de intensidad leve-moderada (1-4 puntos) y con dolor intenso ( $>5$ puntos). Las evaluaciones se llevaron a cabo en dos momentos: inmediato posterior al bloqueo retrobulbar y después de ingresar a la unidad de cuidados posanestésicos. El análisis con la prueba de Kruskal-Wallis reportó diferencias estadísticamente significativas entre los grupos en los dos momentos de medición, con $p=0.003$ y $p=0.000$, respectivamente. De forma similar, el detalle en los reportes de dolor usando escalas tipo Likert para el análisis estadístico con la prueba de ji al cuadrado se muestra en la tabla 4.

Tabla 1. Variables clínico-demográficas de la población en estudio

\begin{tabular}{|c|c|c|c|c|}
\hline & $\begin{array}{c}\text { Grupo } A, \\
\text { gabapentina } 300 \mathrm{mg} \\
(n=41)\end{array}$ & $\begin{array}{c}\text { Grupo } B, \\
\text { gabapentina } 450 \mathrm{mg} \\
(\mathrm{n}=44)\end{array}$ & $\begin{array}{l}\text { Grupo C, } \\
\text { control } \\
(n=40)\end{array}$ & Estadística \\
\hline $\begin{array}{l}\text { Sexo, } n(\%) \\
\text { Femenino } \\
\text { Masculino }\end{array}$ & $\begin{array}{l}18(14.4) \\
23(18.4)\end{array}$ & $\begin{array}{l}43(34.4) \\
82(65.6)\end{array}$ & $\begin{array}{l}13(10.4) \\
27(21.6)\end{array}$ & $x^{2}=2.64 ; g l=2 ; p=0.26$ \\
\hline Edad, años $(X \pm D E)$ & $59.9(13.9)$ & $60.27(13.6)$ & $58.9(11.6)$ & $\mathrm{t}=0.12 ; \mathrm{gl}=2 ; \mathrm{p}=0.886$ \\
\hline$I M C(X \pm D E)$ & $27.4(4.7)$ & $27.2(4.7)$ & $27.3(4.4)$ & $\mathrm{t}=6.90 ; \mathrm{gl}=8 ; \mathrm{p}=0.547$ \\
\hline $\begin{array}{l}\text { Endocrinopatías (\%) } \\
\text { Diabetes mellitus } \\
\text { Hipotiroidismo } \\
\text { Cardiopulmonar (n, \%) } \\
\text { EPOC, SAOS } \\
\text { SICA } \\
\text { Arritmias } \\
\text { Dislipidemia }\end{array}$ & $\begin{array}{c}21(16.8) \\
2(1.6) \\
1(0.8) \\
0(0) \\
2(1.6) \\
1(0.8)\end{array}$ & $\begin{array}{c}27(21.6) \\
1(0.8) \\
2(1.6) \\
2(1.6) \\
0(0) \\
1(0.8)\end{array}$ & $\begin{array}{c}22(17.6) \\
0(0) \\
1(0.8) \\
0(0) \\
0(0) \\
0(0)\end{array}$ & $\begin{array}{l}x^{2}=0.91 ; g l=2 ; p=0.634 \\
x^{2}=3.95 ; g l=4 ; p=0.418 \\
x^{2}=9.38 ; g l=8 ; p=0.317\end{array}$ \\
\hline $\begin{array}{l}\text { Otros, n (\%) } \\
\text { IRC } \\
\text { Tabaquismo } \\
\text { Toxicomanías }\end{array}$ & $\begin{array}{l}2(1.6) \\
9(7.2) \\
1(0.8)\end{array}$ & $\begin{array}{c}7(5.6) \\
12(9.6) \\
1(0.8)\end{array}$ & $\begin{array}{c}4(3.2) \\
18(14.4) \\
2(1.6)\end{array}$ & $\begin{array}{l}x^{2}=2.78 ; g l=2 ; p=0.249 \\
x^{2}=5.49 ; g l=2 ; p=0.064 \\
x^{2}=0.71 ; g l=2 ; p=0.734\end{array}$ \\
\hline
\end{tabular}

DE: desviación estándar; EPOC: enfermedad pulmonar obstructiva crónica; IMC: índice de masa corporal; IRC: insuficiencia renal crónica; SAOS: síndrome de apnea obstructiva del sueño; SICA: síndrome isquémico coronario agudo. 
Tabla 2. Comportamiento hemodinámico y respiratorio de los grupos analizados

\begin{tabular}{|c|c|c|c|c|}
\hline & $\begin{array}{c}\text { Grupo } A, \\
\text { gabapentina } 300 \mathrm{mg} \\
(\mathrm{n}=41) \\
\text { Media (DE) }\end{array}$ & $\begin{array}{c}\text { Grupo } B, \\
\text { gabapentina } 450 \mathrm{mg} \\
(\mathrm{n}=44) \\
\text { Media (DE) }\end{array}$ & $\begin{array}{c}\text { Grupo C, } \\
\text { control } \\
(n=40) \\
\text { Media (DE) }\end{array}$ & Estadística \\
\hline \multicolumn{5}{|l|}{ Frecuencia cardíaca (Ipm) } \\
\hline Basal & $70.0(10.6)$ & $71.5(11.3)$ & $72.9(13.4)$ & $F=0.62 ; g l=2 ; p=0.536$ \\
\hline Transoperatorio & $69.1(10.7)$ & $69.3(11.9)$ & $71.2(11.9)$ & $F=0.40 ; g l=2 ; p=0.666$ \\
\hline Postoperatorio & $65.9(9.3)$ & $67.1(11.2)$ & $69.5(11.7)$ & $F=1.18 ; g l=2 ; p=0.309$ \\
\hline \multicolumn{5}{|l|}{ Presión arterial sistólica (mmHg) } \\
\hline Basal & $152.9(23.6)$ & $152.9(23.6)$ & $149.1(31.2)$ & $F=0.29 ; g l=2 ; p=0.749$ \\
\hline Transoperatorio & $146.7(19.9)$ & $139.8(15.7)$ & $141.5(19.8)$ & $F=1.59 ; g l=2 ; p=0.734$ \\
\hline Postoperatorio & $140.9(19.9)$ & $138.1(18.3)$ & $132.1(27.5)$ & $F=1.66 ; g l=2 ; p=0.193$ \\
\hline \multicolumn{5}{|l|}{ Presión arterial diastólica (mmHg) } \\
\hline Basal & $84.4(10.5)$ & $85.9(10.8)$ & $86.9(9.9)$ & $F=0.56 ; g l=2 ; p=0.567$ \\
\hline Transoperatorio & $80.1(9.1)$ & $78.3(9.1)$ & $80.2(9.5)$ & $F=0.56 ; g l=2 ; p=0.571$ \\
\hline Postoperatorio & $77.4(9.3)$ & $75.5(9.0)$ & $77.9(9.2)$ & $F=0.75 ; g l=2 ; p=0.471$ \\
\hline Presión arterial media (mmHg) & & $86.78(24.7)$ & $85.44(28.6)$ & $F=0.47 ; g l=2 ; p=0.954$ \\
\hline Basal & $87.01(21.2)$ & $73.31(13.5)$ & $76.4(17.7)$ & $F=1.32 ; g l=2 ; p=0.270$ \\
\hline Transoperatorio & $79.05(17.3)$ & $69.65(12.25)$ & $69.34(19.23)$ & $F=0.78 ; g l=2 ; p=0.459$ \\
\hline Postoperatorio & $73.39(17.0)$ & & & \\
\hline \multicolumn{5}{|l|}{ Saturación de oxígeno (\%) } \\
\hline Basal & $92.6(3.6)$ & $94(3.2)$ & $94.0(3.4)$ & $F=2.18 ; g l=2 ; p=0.117$ \\
\hline Transoperatorio & $97.2(1.7)$ & $97.5(1.7)$ & $96.5(2.7)$ & $F=2.62 ; g l=2 ; p=0.076$ \\
\hline Postoperatorio & $95.9(12.9)$ & $97.5(1.8)$ & $97.9(1.5)$ & $F=0.81 ; g l=2 ; p=0.446$ \\
\hline \multicolumn{5}{|l|}{ Frecuencia respiratoria (rpm) } \\
\hline Basal & $16.4(1.2)$ & $16.5(1.0)$ & $16.4(0.9)$ & $F=0.076 ; g l=2 ; p=0.927$ \\
\hline Transoperatorio & $16.6(1.3)$ & $15.9(1.7)$ & $16.2(1.0)$ & $F=2.30 ; g l=2 ; p=0.104$ \\
\hline Postoperatorio & $16.1(1.5)$ & $16.2(1.1)$ & $16.1(0.8)$ & $F=0.072 ; g l=2 ; p=0.930$ \\
\hline
\end{tabular}

Tabla 3. Grado de ansiedad Likert en los grupos

\begin{tabular}{|c|c|c|c|c|}
\hline & $\begin{array}{c}\text { Grupo A, gabapentina } 300 \mathrm{mg} \\
\mathrm{n}(\%)\end{array}$ & $\begin{array}{c}\text { Grupo B, gabapentina } 450 \mathrm{mg} \\
\mathrm{n}(\%)\end{array}$ & $\begin{array}{c}\text { Grupo } \mathrm{C} \text {, control } \\
\mathrm{n}(\%)\end{array}$ & Estadística \\
\hline Preoperatorio & & & & $x^{2}=6.92 ; g l=6 ; p=0.328$ \\
\hline Nada & $3(2.4)$ & $4(3.2)$ & $1(0.8)$ & \\
\hline Poca & $21(16.8)$ & $14(11.2)$ & $15(12.8)$ & \\
\hline Moderada & $12(9.6)$ & $22(17.6)$ & $16(12.8)$ & \\
\hline Fuerte & $5(4.0)$ & $4(3.2)$ & $7(5.6)$ & \\
\hline Transoperatorio & & & & $x^{2}=17.25 ; g l=6 ; p=0.008$ \\
\hline Nada & $23(18.4)$ & $12(9.6)$ & $9(7.2)$ & \\
\hline Poca & $16(12.8)$ & $22(17.5)$ & $19(15.2)$ & \\
\hline Moderada & $2(1.6)$ & $9(7.2)$ & $12(9.6)$ & \\
\hline Fuerte & $0(0)$ & $1(0.8)$ & $0(0)$ & \\
\hline Postoperatorio & & & & $x^{2}=15.66 ; g l=6 ; p=0.016$ \\
\hline Nada & $32(25.6)$ & $23(18.4)$ & $16(12.8)$ & \\
\hline Poca & $8(6.4)$ & $21(16.8)$ & $22(17.6)$ & \\
\hline Moderada & $1(0.8)$ & $0(0)$ & $1(0.8)$ & \\
\hline Fuerte & $0(0)$ & $0(0)$ & $1(0.8)$ & \\
\hline
\end{tabular}

El consumo de total de fentanilo durante el procedimiento quirúrgico fue de $98.17 \pm 30.8 \mu \mathrm{g}$ (grupo A), $107.05 \pm 26.8 \mu \mathrm{g}$ (grupo B) y $119.32 \pm 35.70 \mu \mathrm{g}$ (grupo $\mathrm{C}$ ), con diferencias estadísticamente significativas $(F=3.80 ; g l=2 ; p=0.025)$.

\section{Discusión}

En nuestro estudio, el uso de gabapentina para aminorar la presencia de hipertensión reactiva no demostró diferencia clínica ni estadísticamente significativa en 
Tabla 4. Reporte de dolor de los grupos analizados

\begin{tabular}{lcccc}
\hline & $\begin{array}{c}\text { Grupo A, } \\
\text { gabapentina } \mathbf{3 0 0} \mathbf{~} \mathbf{g}\end{array}$ & $\begin{array}{c}\text { Grupo B, } \\
\text { gabapentina } \mathbf{4 5 0} \mathbf{~ m g}\end{array}$ & $\begin{array}{c}\text { Grupo C, } \\
\text { control }\end{array}$ & Estadística \\
\hline $\begin{array}{l}\text { Bloqueo retrobulbar, } \mathrm{n}(\%) \\
\text { Ausente }\end{array}$ & $34(27.2)$ & $28(22.4)$ & $16(12.8)$ & \\
Leve-moderado & $7(5.6)$ & $14(11.2)$ & $17(13.6)$ & \\
Intenso & $0(0)$ & $2(1.6)$ & $7(5.6)$ & \\
UCPA, $\mathrm{n}(\%)$ & & & & \\
Ausente & $40(32)$ & $37(29.6)$ & $23(18.4)$ & \\
Leve-moderado & $1(0.8)$ & $6(4.8)$ & $17(13.6)$ & \\
Intenso & $0(0)$ & $1(0.8)$ & $0(0)$ & \\
\hline
\end{tabular}

UCPA: unidad de cuidados posanestésicos.

pacientes operados de cirugía oftalmológica. Así mismo, con las dosis aplicadas no parecen presentarse modificaciones importantes en el comportamiento hemodinámico ni respiratorio antes y después de la cirugía, lo que concuerda con publicaciones previas en las que la gabapentina ha sido utilizada como premedicación en cirugías de catarata, a dosis mucho mayores $(1200 \mathrm{mg}$ ), aunque en el estudio de Azeem y Marey ${ }^{27}$, si bien hubo una tendencia a cifras tensionales más bajas en el grupo de gabapentina, no se encontraron diferencias estadísticamente significativas. Del mismo modo, en un ensayo clínico controlado en el que se usó gabapentina $(900 \mathrm{mg})$ como premedicación para la cirugía de nariz y laringe no se observaron modificaciones significativas en el comportamiento hemodinámico ni respiratorio, situación similar a la obtenida en nuestro estudio28. Tampoco pudimos encontrar razón alguna para que existiera una tendencia a cifras tensionales mayores en los grupos que usaron gabapentina. Dado lo anterior, es posible que las dosis empleadas fueron insuficientes para aminorar la respuesta simpática que se refleja en un incremento transitorio de las presiones arteriales como consecuencia de la ansiedad que se presenta durante el preoperatorio.

La hipertensión reactiva es una respuesta secundaria a episodios de ansiedad ${ }^{29}$, por lo que la evaluación de la ansiedad preoperatoria fue otra variable fundamental a medir. Para la medición del grado de ansiedad planteamos utilizar el cuestionario estandarizado de la escala APAIS, una escala frecuentemente utilizada en diversos estudios de ansiedad perioperatoria; sin embargo, no se encuentra validada al español ${ }^{30}$ y por ello utilizamos escalas tipo Likert. Por otro lado, Ménigaux, et al. ${ }^{31}$ demostraron en un ensayo clínico controlado que el uso de gabapentina en el preoperatorio produce una disminución estadísticamente significativa de la ansiedad. También se han reportado beneficios como ansiolítico en trastornos psiquiátricos tales como ataques de pánico ${ }^{27}$. No obstante, al momento no se han descrito los mecanismos precisos por los cuales la gabapentina ejerce su efecto ansiolítico; una posible vía es el aumento de la síntesis del neurotransmisor GABA por enzimas reguladoras ${ }^{32}$. En este estudio no parece haber un efecto ansiolítico mayor con dosis superiores de gabapentina cuando se comparan 300 y 450 mg, pero sí con respecto al grupo control, por lo que puede iniciarse la búsqueda de dosificaciones más óptimas para premedicación en el manejo de la ansiólisis perioperatoria, máxime en nuestra población, dado que no se ha explorado en nuestro medio, y más aún por los múltiples efectos adversos que se presentan frecuentemente como consecuencia de la medicación cuanto mayor es la dosis empleada.

En otro contexto, mucho se ha cuestionado sobre el mecanismo analgésico de la gabapentina y se ha concluido que inhibe la transmisión neural por los canales de calcio dependientes del voltaje, inhibiendo la liberación de neurotransmisores excitatorios ${ }^{33,34}$.

El uso de $1.2 \mathrm{~g}$ de gabapentina una hora antes de la cirugía disminuye las cifras reportadas de dolor posoperatorio, así como el consumo de opiáceos durante la cirugía ${ }^{33}$. Peng, et al. ${ }^{35}$ concluyeron en un metaanálisis que su uso en el perioperatorio disminuye el consumo total de opiáceos y potencia sus efectos analgésicos, traduciéndose como una disminución importante del dolor agudo posoperatorio. En nuestro estudio, la premedicación con gabapentina demostró ser de utilidad en el control de dolor durante el transanestésico y el posanestésico, reportando cifras inferiores en la percepción de dolor por parte de los grupos en que se administró gabapentina comparados con el grupo control, con un mayor consumo de opiáceos en este, y con diferencias estadísticamente significativas. Los autores consideramos que una contribución importante del estudio es reportar que dosis de $300 \mathrm{mg}$ son suficientes para disminuir los 
requerimientos totales de opiáceos y los reportes de ansiedad en el perioperatorio, además de que estas respuestas son similares a las obtenidas cuando se usan dosis de $450 \mathrm{mg}$.

Si bien no se logró demostrar un efecto particular de la gabapentina sobre la hipertensión reactiva, sí se vieron sus efectos sobre el estado de ansiedad y la analgesia sin reporte de efectos secundarios importantes, a excepción de somnolencia leve.

\section{Conclusiones}

El régimen de administración por vía oral de 3000 $450 \mathrm{mg}$ de gabapentina 2 horas antes de la cirugía reduce el dolor y la ansiedad durante el posoperatorio inmediato, así como el consumo de opiáceos, aunque no disminuye la hipertensión reactiva asociada a estos factores en los pacientes sometidos a cirugía oftalmológica.

\section{Bibliografía}

1. Rosenman $\mathrm{RH}$. Does anxiety or cardiovascular reactivity have a causal role in hypertension? Integr Physiol Behav Sci. 1991;26:296-304.

2. Goldmann L, Ogg TW, Levey AB. Hypnosis and day case anaesthesia. A study to reduce pre-operative anxiety and intraoperative anaesthetic requirements. Anaesthesia. 1988;43:466-9.

3. Maranets I, Kain ZN. Pre-operative anxiety and intra operative anesthetic requirements. Anesthesia and Analgesia.1999;89:1346-51

4. Caumo W, Schmidt AP, Schneider CN, et al. Risk factors for pre-operative anxiety in adults. Acta Anaesthesiologica Scandinavica. 2001;45:298-307.

5. Londsdale M, Hutchison GL. Patients desire for information about anaesthesia: Scottish and Canadian attitudes. Anaesthesia. 1991;46: 410-2

6. Thomas T, Robinson C, Champion D, et al. Prediction and assessment of the severity of post-operative pain and of satisfaction with management. Pain. 1998;75:177-85.

7. Al-Mujadi H, A-Refai AR, Katzarov MG, et al. Preemptive gabapentin reduces postoperative pain and opioid demand following thyroid surgery. Can J Anesth. 2006;53:268-73

8. Thomas V, Heath M, Rose D, et al. Psychological characteristics and the effectiveness of patient-controlled analgesia. $\mathrm{Br} \mathrm{J}$ Anaesth. 1995;74: 271-6.

9. Moix J. Emoción y cirugía. An Psicol. 1994;10:111-8.

10. Izurieta R, Jeffrey TR. Sedation during mechanical ventilation: a systematic review. Crit Care Med. 2002;30:118-25.

11. L'Abbé LA, Detsky AS, O'Rourke K. Meta-analysis in clinical research. Ann Intern Med. 1987;107:224-33.

12. Wetsch WA, Lederer W, Kinz F, et al. Preoperative stress and anxiety in day-care patients and in patients undergoing fast-track surgery. $\mathrm{Br} \mathrm{J}$ Anaesth. 2009;103:199-205
13. Busch JA, Radulovic LL, Bockbrader HN. Effect of Maalox TC on single-dose pharmacokinetics of gabapentin capsules in healthy subjects. Pharm Res. 1992;9:315

14. Pollack MH, Matthews J, Scott EL. Gabapentin as a potential treatment for anxiety disorders. Am J Psychiatry. 1998;155:992-3.

15. Turan A, Karamanlioglu B, Memis D, et al. The analgesic effects of gabapentin after total abdominal hysterectomy. Anesth Analg. 2004;98:1370-3

16. Turan A, Memis D, Karamanlioglu B, et al. The analgesic effects of gabapentin in monitored anesthesia care for ear-nose-throat surgery. Anesth Analg. 2004;99:375-8

17. Gilron I, Biederman J, Jhamandas K, et al. Gabapentin blocks and reverses antinociceptive morphine tolerance in the rat paw pressure and tail-flick tests. Anesthesiology. 2003;98:1288-92.

18. Kong VK. Irwin MG. Gabapentin: a multimodal perioperative drug? $\mathrm{Br} \mathrm{J}$ Anaesth. 2007:99:775-86.

19. Tirault M, Foucan M, Debaene B, et al. Gabapentin premedication: assessment of preoperative anxiolysis and postoperative patient satisfaction. Acta Anaesth. 2010;61:203-9.

20. Fassoulaki A, Triga $A$, Melemeni $A$, et al. Multimodal analgesia with gabapentin and local anesthetics prevents acute and chronic pain after breast surgery for cancer. Anesth Analg. 2005;101:1427-32.

21. Rickels $\mathrm{K}$, Pollack MH, Feltner DE, et al. Pregabalin for treatment of generalized anxiety disorder. A 4-week, multicenter, double-blind, placebo-controlled trial of pregabalin and alprazolam. Arch Gen Psychiary. 2005;62:1022-30.

22. Tipanna EM, Hamunen K, Kontinen VK, et al. Do surgical patients benefit from perioperative gabapentin/pregabalin? A systematic review of efficacy and safety. Anesth Analg. 2007:104:1545-56.

23. Adam F, Ménigaux C, Sessler DI, et al. A single preoperative dose of gabapentin (800 milligrams) does not augment postoperative analgesia in patients given interscalene brachial plexus blocks for arthroscopic shoulder surgery. Anesth Analg. 2006:103:1278-82.

24. Bonhaus DW, Loo C, Secchi R, et al. Effects of the GABAB receptor antagonist CGP 55845 on the anticonvulsant and anxiolytic actions of gabapentin. Pharmacologist. 2002;44:100.

25. Seib RK, Paul JE. Preoperative gabapentin for postoperative analgesia: a meta-analysis. Can J Anesth. 2006;53:461-9.

26. Montazeri K, Kashefi P, Honarmand A. Pre-emptive gabapentin significantly reduces postoperative pain and morphine demand following lower extremity orthopaedic surgery. Med J. 2007;48:748.

27. Azeem $\mathrm{E}$, Marey $\mathrm{H}$. The effect of gabapentin premedication on pain and anxiety during cataract surgery under peribulbar block. Egyptian Journal of Anesthesia. 2012;28:43-7.

28. Farzi F, Haddadi S, Ebrahimpour N, et al. A survey on the effect of oral gabapentin on hemodynamic changes during direct laryngoscopy and tracheal intubation and intraoperative bleeding in patients undergoing septorhinoplasty. Anesth Pain Med. 2015;5:29705.

29. Susan A, Everson JW, Lynch GA, et al. Stress-induced blood pressure reactivity and incident stroke in middle-aged men. Stroke. 2001;32:1263-70

30. Doñate-Marín D, Litago-Cortés A, Monge-Sanz $\mathrm{Y}$, et al. Aspectos de la información preoperatoria relacionada con la ansiedad del paciente programado para cirugía. Enfermeria Global. 2015;37:170-80.

31. Ménigaux C, Adam F, Guignard B, et al. Preoperative gabapentin decreases anxiety and improves early functional recovery from knee surgery. Anesth Analg. 2005;100:1394-9.

32. Chouinard G, Beauclair L, Belanger MC. Gabapentin: long-term antianxiety and hypnotic effects in psychiatric patients with comorbid anxiety-related disorders. Can J Psychiatry. 1998;43:305.

33. Chang CY, Challa CK, Shah J, et al. Gabapentin in acute postoperative pain management. Biomed Res Int. 2014;2014:631756.

34. Melemeni A, Staikou C, Fassoulaki A. Gabapentin for acute and chronic post-surgical pain. Signa Vitae 2. Pharmamed. 2007:1:42-51.

35. Peng PW, Wijeysundera DN, Li CC. Use of gabapentin for perioperative pain control: a meta-analysis. Pain Res Manag. 2007;12:85-92. 